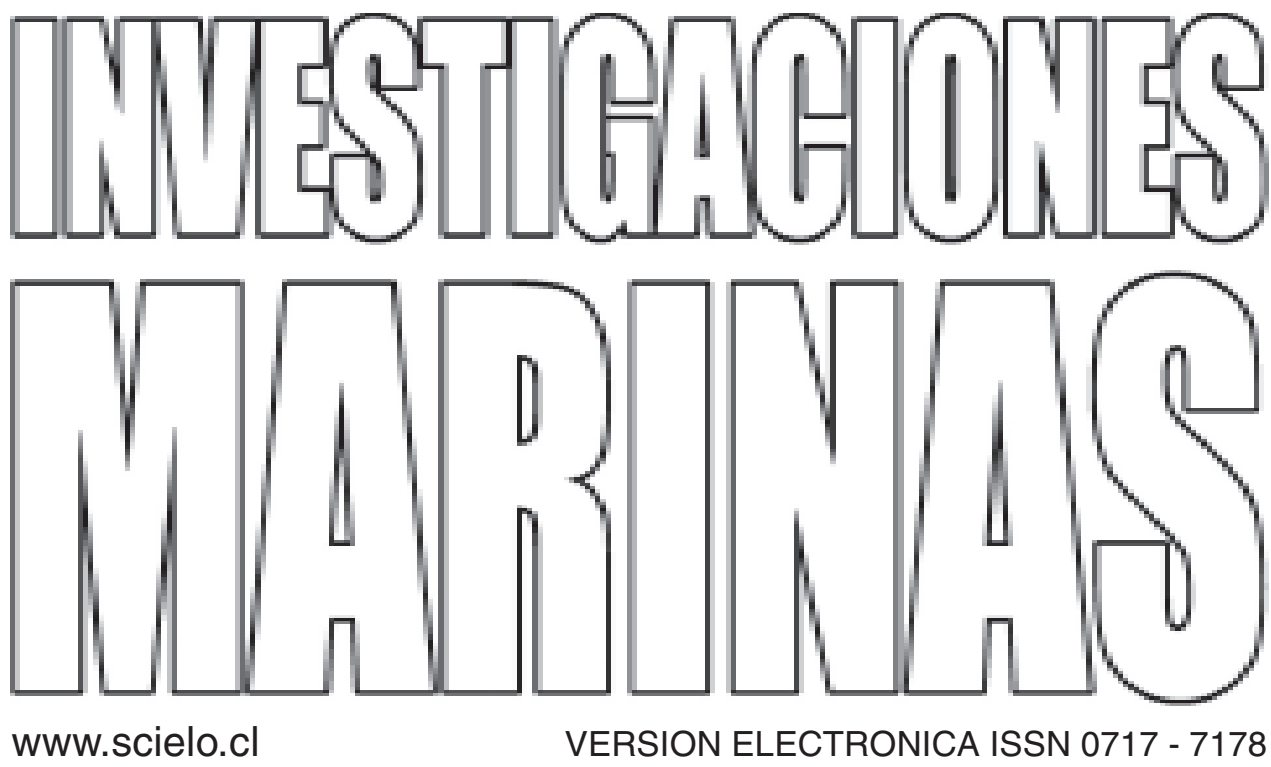

INVESTIGACIONES MARINAS es una revista que considera para su publicación los resultados de investigaciones científicas y tecnológicas originales en ciencias del mar, dando preferencia a los trabajos realizados en el Océano Pacífico Sudoriental y Océano Austral. Los artículos y notas pueden ser publicados en idioma español o inglés.

La revista es de periodicidad semestral y es editada por la Escuela de Ciencias del Mar de la Facultad de Recursos Naturales de la Pontificia Universidad Católica de Valparaíso.

INVESTIGACIONES MARINAS is a journal for the purpose of publishing results either from scientific or technological research in marine science. The journal will give priority to those manuscripts referring to the Southeastern Pacific or the Southern Ocean. Submission can be made in Spanish or English.

The journal is edited by Escuela de Ciencias del Mar, Facultad de Recursos Naturales, Pontificia Universidad Católica de Valparaíso, and published twice a year.

PONTIFICIA UNIVERSIDAD CATÓLICA DE VALPARAÍSO

FACULTAD DE RECURSOS NATURALES

ESCUELA DE CIENCIAS DEL MAR 


\section{EVALUADORES DE LOS TRABAJOS INCLUIDOS EN ESTE NÚMERO}

\author{
Ramón Ahumada \\ Universidad Católica de la Santísima Concepción \\ Hugo Arancibia \\ Universidad de Concepción \\ Pedro Báez \\ Museo Nacional de Historia Natural \\ Cristian Canales \\ Instituto de Fomento Pesquero \\ Enrique Dupré \\ Universidad Católica del Norte \\ Jaime Färber \\ Centro de Investigación Científica de Educación \\ Superior de Ensenada, México \\ Guillermo Guzmán \\ Universidad Arturo Prat \\ Julio Lamilla \\ Universidad Austral de Chile
}

Roberto Meléndez

Museo Nacional de Historia Natural

Sergio Navarrete

Pontificia Universidad Católica de Chile

Ignacio Payá

Instituto de Fomento Pesquero

Jaime Pizarro

Universidad de Santiago de Chile

Marco Salamanca

Universidad de Concepción

Walter Sielfeld

Universidad Arturo Prat
Tarsicio Antezana

Universidad de Concepción

Dagoberto Arcos

Instituto de Investigación Pesquera

Sophie Bertrand

Institut de Recherche pour le Developpement, Francia

Juan Carlos Castilla

Pontificia Universidad Católica de Chile

Rubén Escribano

Universidad de Concepción

Humberto González

Universidad Austral de Chile

Ismael Kong

Universidad de Antofagasta

Bertha Lavaniegos

Centro de Investigación Científica de Educación Superior de Ensenada, México

Carlos Moreno

Universidad Austral de Chile

Francesc Pagès

Instituto de Ciencias del Mar de Barcelona, España

Mario Pino

Universidad Austral de Chile

Ricardo Prego

Instituto de Investigaciones Marinas, Vigo, España

Marcelo Scelzo

Universidad Nacional de Mar del Plata, Argentina

Raúl Soto

Universidad Arturo Prat 


\title{
Especiación de zinc en sedimentos marinos del fiordo Aysén
}

\author{
Ramón Ahumada ${ }^{1}$, Elizabeth González ${ }^{1} \&$ José Neira $^{2}$ \\ ${ }^{1}$ Universidad Católica de la Santísima Concepción, Facultad de Ciencias, Casilla 297, Concepción \\ ${ }^{2}$ Universidad de Concepción, Facultad de Farmacia, Casilla 237, Concepción \\ E-mail: rahuma@ucsc.cl; eligoza@ucsc.cl; vneira@udec.cl
}

\begin{abstract}
RESUMEN. Se analiza la especiación de zinc presente en los sedimentos superficiales del fiordo Aysén mediante un ataque diferencial para identificar la dominancia de las diferentes especies de Zn. Las muestras se obtuvieron en dos estaciones, una en la boca y la otra en la cabeza del fiordo, en noviembre de 2001. Los resultados de zinc total fueron de $168,0 \pm 15 \mu \mathrm{g} \cdot \mathrm{g}^{-1} \mathrm{en}$ la cabeza (correspondiendo a sedimentos enriquecidos en $\mathrm{Zn}$ ) y $83,6 \pm 6 \mu \mathrm{g} \cdot \mathrm{g}^{-1} \mathrm{en} \mathrm{la} \mathrm{boca} \mathrm{del}$ fiordo (concentración levemente inferior al valor de la línea de base). Estos resultados fueron consistentes con las concentraciones de zinc total determinados en 1995, en estaciones de similar ubicación geográfica. El estudio de especiación de Zn, mostró que en la cabeza del fiordo, dominan en abundancia las concentraciones de las fracciones: residual $>$ oxihidróxidos $>$ intercambiables $>$ materia orgánica $>$ carbonatos. En cambio, en la boca, dominan la fracción residual $>$ oxihidróxidos ${ }^{3}$ intercambiables $>$ carbonatos $>$ materia orgánica. Respecto del análisis de las concentración, entre los valores absolutos de las especies de $\mathrm{Zn}$ en los sedimentos, estas resultaron ser el doble en Puerto Chacabuco (puerto de embarque de concentrado de mineral), en relación a la boca de fiordo Aysén. Las proporciones de cada una de las fracciones entre los sitios estudiados fueron: intercambiables : carbonatos : oxihidróxidos : materia orgánica : residuales $=2,7: 0,4: 11,2: 7,5: 1,4$. No obstante, si se toman los porcentajes de las diferentes especies sobre la concentración total, para cada uno de los sitios, se observó que el mayor porcentaje de la fracción residual ocurre en la boca del fiordo.
\end{abstract}

Palabras claves: especiación química, sedimentos marinos, espectrofotometría de absorción atómica, fiordos, Aysén, Chile.

\section{Zinc speciation in marine sediments of Aysén Fjord}

\begin{abstract}
Zinc speciation in marine shallow sediments from Aysen fjord was analyzed using a differential attack. This treatment was used in order to identify the dominance of the different $\mathrm{Zn}$ species. Samples were collected in two stations, one of them in the fjord mouth, and the other in the fjord head, november 2001. The Zn total results were: $168.0 \pm 15 \mu \mathrm{g} \cdot \mathrm{g}^{-1}$ at the fjord head (corresponding to enriched sediments) and, $83.6 \pm 6 \mu \mathrm{g} \cdot \mathrm{g}^{-1}$ at the fjord mouth (concentration close to base line value in this area). These results were consistent with the $\mathrm{Zn}$ total concentration from the samples obtained in 1995, in stations placed at the same geographic location. The study of speciation of $\mathrm{Zn}$ showed that at Stn. 82-a the metals, in order of abundance, were associated with the residual fraction $>$ oxyhydroxides $>$ interchangeables $>$ organic matter $>$ carbonates. In contrast, at Stn. 78-a, in order of abundance the residual fraction $>$ oxyhydroxides ${ }^{3}$ interchangeables $>$ carbonates $>$ carbonates $>$ organic material. The resultant relationship among the species of the interchangeable sites were: carbonates : oxyhydroxides $:$ organic material $:$ residuals $=2.7: 0.44: 11.2$ $: 7.5: 1.4$. The percentages of all the species were interchangeable: carbonates : oxyhydroxides : organic material : residuals $=1.37: 0.21: 5.73: 3.9: 0.71$. These results showed that the absolute values for concentrations were independent of percentages of the species. The relationship between, concentration in the sediments showed that Puerto Chacabuco (shipment site of concentrate minerals), had twice the Zn concentrations shown by samples from the mouth of Aysén Fjord.
\end{abstract}

Key words: chemical speciation, marine sediments, atomic absorption spectrometry, fjords, Aysén, Chile. 


\section{INTRODUCCIÓN}

La especiación química se ha definido como el proceso de identificación y cuantificación de diferentes especies, formas o fases químicas presentes en una matriz ambiental (Davidson et al., 1994). Los sitios sedimentarios durante su formación se ordenan en estratos superpuestos, dejando un registro de los compuestos químicos que entran a los ambientes acuáticos. Cualquier entrada adicional de metales a la columna de agua quedará registrada en los sedimentos, a través de la fracción de metales que reacciona con el material particulado y coloidal al sedimentar (Wen et al., 1999). Por sus características dinámicas, los sedimentos son un resumidero de los diferentes compuestos químicos y por tanto, son una matriz compleja y heterogénea. No todas las fracciones de metales que forman parte del sedimento permanecen, hay fracciones que reaccionan por cambios en las condiciones del medio, pudiendo liberarse y constituir un riesgo potencial para los organismos bentónicos (Tessier \& Campbell, 1988).

Los metales traza contenidos en los sedimentos marinos de zonas remotas (escasa densidad poblacional), provienen principalmente de la erosión de las fases detríticas del basamento rocoso de la región, a través de los aportes fluviales, atmosféricos o advectivos. La actividad antropogénica modifica la concentración natural a escala local.

Los estudios de abundancia de metales en sedimentos marinos realizados hasta ahora en la zona de fiordos, estaban referidos al contenido total de algunos metales (Ahumada, 1998, 1999; 2001; Pineda et al., 2002). La extracción secuencial es un método que se utiliza para estudiar la especiación de los metales en el sedimento, y permite cuantificar la biodisponibilidad de los contaminantes asociados a sedimentos o suelos (Howard \& Sledzinski, 1996; Tessier, 1999), estimar el comportamiento de los elementos trazas involucrados en los procesos autogénicos (Wen et al., 1999) y establecer las tasas de removilización durante la diagénesis temprana o resuspensión de los elementos depositados en los sedimentos (Martin et al., 1987; Rivera-Duarte \& Flegal, 1997). Esta técnica se ha usado también para establecer la dinámica de los procesos químicos y geoquímicos que ocurren en la interfase aguasedimento, neoformación de minerales, removilización de metales traza y/o para modelar el comportamiento de distintas especies químicas (Luoma \& Davis, 1983; Warnken et al., 2001).
El fiordo Aysén está ubicado en la región norte de los canales del sur de Chile (4520's; $73^{\circ} 05^{\prime} \mathrm{W}$ ) y está conectado indirectamente a la costa del Pacífico. Es un fiordo con un régimen de estuario altamente estratificado (Pickard, 1973; Silva et al., 1997). Presenta un fondo con tres cuencas y una explanada sedimentaria del postglaciar de material reciente producto del deslizamiento de las vertientes (Araya, 1997). En la cabeza del fiordo Aysén se localiza Puerto Chacabuco, que desde 1960 se utiliza como un puerto de embarque de concentrado de Zn y Pb. Entre 1990 y 2001 se embarcaron, 344.894 ton de concentrado de $\mathrm{Zn}$ y 8.121 ton de concentrado de $\mathrm{Pb}$, proveniente de la Compañía Minera “El Toqui".

Antecedentes obtenidos en el fiordo Aysén en el estudio de la distribución del contenido $\mathrm{Zn}$ total $\left(\mathrm{Zn}_{\text {Total }}\right)$ en los sedimentos (Ahumada, 1998), muestran un patrón de distribución vertical con un máximo en superficie y un gradiente negativo en los primeros $15 \mathrm{~cm}$ para estabilizarse en los $15 \mathrm{~cm}$ siguientes. Los valores máximos determinados en la superficie de testigos de sedimentos de $30 \mathrm{~cm}$, alcanzaron dos veces la concentración del metal en la base. Además, se observó un gradiente de concentración en los sedimentos superficiales respecto a la boca del fiordo (Ahumada, 1998). El máximo de concentración de Zn detectado en la estación ubicada cerca de Puerto Chacabuco disminuyó hacia la boca del fiordo hasta alcanzar un valor de $86 \mu \mathrm{g} \cdot \mathrm{g}^{-1}$ (Ahumada, 1998) considerado como un valor propio del basamento rocoso de la zona.

El incremento de Zn total en Puerto Chacabuco está asociado a las faenas de embarque de concentrado. A pesar que no puede descartarse el posible transporte de $\mathrm{Zn}$ por el río Aysén, que recibe aguas del río Mañihuales, que proviene de las cercanías de las faenas mineras que se desarrollan a casi 120 $\mathrm{km}$.

El enriquecimiento de Zn total en los sedimentos de la cabeza, en relación a las concentraciones en la boca del fiordo, motivó realizar un estudio de especiación de Zn como una forma de detectar las especies de Zn de mayor biodisponibilidad. Además, se establecieron y cuantificaron las fracciones de Zn que podrían estar intercambiándose entre el sedimento y la columna de agua en una zona enriquecida. 


\section{MATERIALES Y MÉTODOS}

El muestreo se realizó durante el Crucero Cimar 7 Fiordos a bordo del AGOR "Vidal Gormaz", en noviembre de 2001. Durante este crucero se repitió la posición de las estaciones muestreadas en el Crucero Cimar 1 Fiordos (1995) en el fiordo Aysén (Estaciones 82 y 77) (Fig. 1). Para diferenciar las muestras de las del primer crucero, se denominaron Est. 82-a y Est. 78-a respectivamente. Las muestras de sedimentos marinos superficiales se obtuvieron con un box Corer de 30 × 30 × $30 \mathrm{~cm}$ de acero inoxidable. El sedimento se submuestreó con tres testigos de PVC de $6 \mathrm{~cm}$ de diámetro y $30 \mathrm{~cm}$ de longitud, y se almacenaron herméticamente a $4^{\circ} \mathrm{C}$. La fracción de los primeros $2 \mathrm{~cm}$ de sedimentos de cada testigo se destinó al análisis de los sedimentos superficiales. En el laboratorio, el sedimento se secó a peso constante, disgregó en un mortero de ágata y homogeneizó bajo campana de flujo laminar. Para el estudio de especiación se utilizó el método de extracción parcial, una lixiviación controlada de un gramo de muestra, mediante el esquema de Tessier \& Campbell (1988) y Martin et al. (1987), para cinco fracciones del zinc ligado a intercambiables, carbonatos, óxidos e hidróxidos, materia orgánica y residuales (Anexo 1).

El análisis se realizó en un espectrofotómetro de absorción atómica, modelo GBC 902 con un tubo en la llama (TS-FF-AAS) (Neira \& Berndt, 2000). La calibración del equipo y el establecimiento de linealidad se realizó con patrones de Zn preparados a partir de un titrisol Merck mediante una curva de calibración $\left(r^{2}=0,99\right)$, con la técnica de adición de estándares.

Las determinaciones analíticas de las muestras se realizaron por adición estándar múltiple (cuatro adiciones). El cálculo del porcentaje de error relativo (Eurochem, 2001) se realizó utilizando material de referencia certificado "CASS-3" (agua de mar) y "MESS-2" (sedimento marino) (Tabla 1).

\section{RESULTADOS}

El sedimento de las áreas estudiadas correspondió a limo arcilla (diámetro $<0,65 \mu \mathrm{m}$ ) en ambas estaciones, al igual que en el Crucero Cimar 1 Fiordos. Las concentraciones de $\mathrm{Zn}_{\text {Total }}$ en los sedimentos fueron de $168 \pm 15 \mu \mathrm{g} \cdot \mathrm{g}^{-1}$ en la estación 82-a, frente a Puerto Chacabuco y $83,6 \pm 6 \mu \mathrm{g} \cdot \mathrm{g}^{-1}$ para la estación 78-a, en la boca del fiordo. Al comparar las concentraciones con sus estaciones equivalentes del Cimar 1 Fiordos (Ahumada, 1998), se observó semejanzas (Tabla 2). Debe considerarse que los valores de $\mathrm{Zn}_{\text {Total }}$ se obtuvieron mediante técnicas analíticas distintas i.e., con espectrofotometría de Emisión Atómica con Acoplamiento Inductivo de Plasma (SAE-ICP) en las muestras del Cimar 1 Fiordos y con espectrofotometría de Absorción Atómica con tubo en la llama (TS-FF-AAS) en el Cimar 7 Fiordos.

Las concentraciones de las especies de $\mathrm{Zn}$ se presentan en la Tabla 3 como porcentaje del $\mathrm{Zn}_{\text {Total }}$, con el objeto de comparar los procesos y como con-

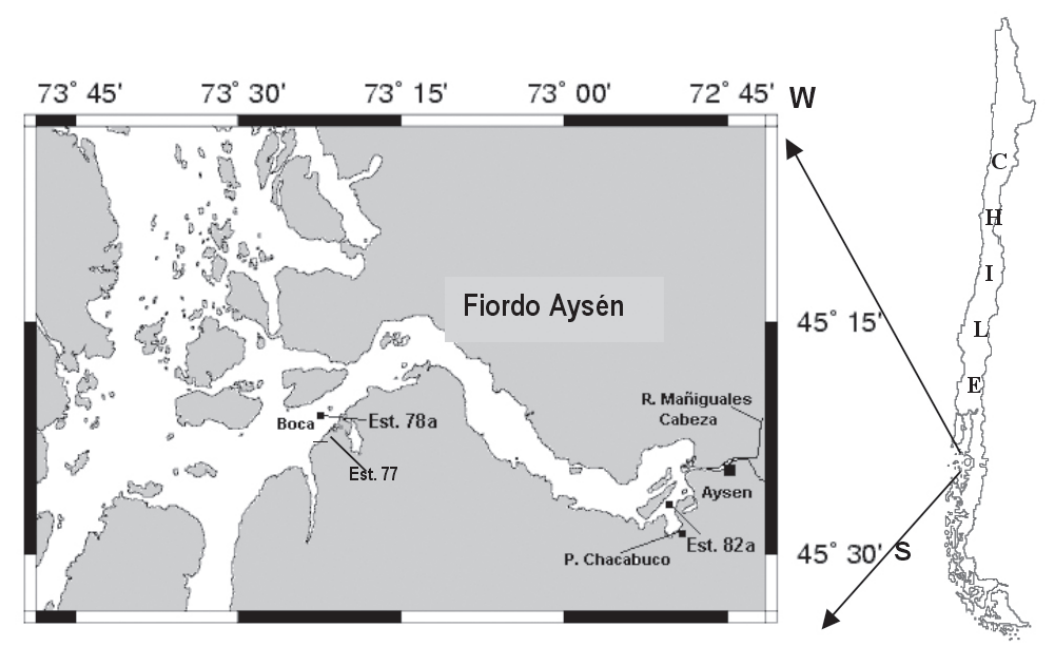

Figura 1. Localización geogrática de las estaciones de muestreo en el fiordo Aysén.

Figure 1. Geographic location of the sampling stations in Aysén Fjord. 
centración nominal como una forma de mostrar las diferencias de concentración entre los sitios, y evaluar las posibles entradas de $\mathrm{Zn}$ al sistema.

El coeficiente de variación de los análisis fluctuó entre 4,8\% para intercambiables y 6,3\% para carbonatos. Esto indicaría que el análisis químico fue realizado con una buena precisión y repetibilidad, en consideración a la manipulación realizada para el análisis de las fracciones. El mayor coeficiente de variación se registró en la fracción de Zn asociado a materia orgánica.

\section{DISCUSIÓN}

El método de extracción secuencial ha generado críticas por limitaciones relacionadas al hecho de ser un método operacional, y a posibles problemas que podrían generarse por las reacciones secundarias de los metales, durante los cambios de condiciones químicas en el proceso de análisis (Kim \& Fergunsson, 1991). Se ha buscado controlar las reacciones de reabsorción de los metales a través de agentes acomplejantes (Howard \& Sledzinski, 1996; Howard \& Vandenbrink, 1999). Sin embargo, los resultados publicados muestran que los problemas de reabsorción y reactividad, ocurren en niveles altos de contaminación (Tessier, 1999). Por tanto, en sitios remotos donde el enriquecimiento es sólo el doble en magnitud de los valores de línea base, esta aplicación metodológica se puede considerar aceptable.

La elección de la técnica analítica de TS-FF-AAS se debió a que se logra un mayor incremento de sensibilidad (i.e. $10^{-8}$ ) y a que es una técnica analítica específica.

Los sitios analizados se seleccionaron en función del área enriquecida (cabeza del fiordo) en relación a un área de referencia (boca del fiordo) distante a $60 \mathrm{~km}$. Ambos sitios presentan características sedimentarias e hidrográficas semejantes, pues corresponden a sitios sedimentarios profundos (Araya, 1997), con condiciones hidrográficas semejantes en esas profundidades (Silva et al., 1997).

El riesgo ambiental se evaluó en función de la concentración Zn en las fracciones de mayor labilidad en el sedimento. De tal manera, que la fracción residual de difícil remoción en la naturaleza, se considera que no representaría un riesgo ambiental. En cambio, el $\mathrm{Zn}$ enlazado a las fracciones intercambiables y carbonatos, presenta un riesgo ambiental mayor dependiendo de su concentración, ya que, debido a su labilidad puede ser removilizado. Es importante constatar que la fracción de intercambiables es semejante en porcentaje en ambas estaciones, lo que estaría de acuerdo con que una pequeña fracción del total en condiciones naturales, reacciona formando enlaces débiles y otra se enlaza a los carbonatos. La fracción de $\mathrm{Zn}$ asociada a carbonatos es mayor en la Est. 78-a, boca del fiordo que en la cabeza. Esta condición puede sustentarse en una mayor concentración de los carbonatos sedimentarios por el aporte de tecas de foraminíferos bentónicos, donde la preservación de los carbonatos puede explicarse por menor concentración de la materia orgánica y mayor dinámica del sistema (Libes, 1992).

En esta área, las fracciones que pueden ser removilizadas son las fracciones intercambiables y carbonatos, que corresponden a un 7,6\% del $\mathrm{Zn}$ total en la estación 82-a y un 8,8\% de Zn total en la estación 78-a. Este resultado expresado como porcentaje del total aparece como contradictorio, debido a que la mayor fracción de Zn removilizable proviene de la Est. 78-a, que corresponde a la estación de referencia. En cambio, en términos de concentración, la fracción de Zn removilizable en la Est 78-a fue solo de 7,4 $\mu \mathrm{g} \cdot \mathrm{g}^{-1}$, mientras que en la Est. 82-a (enriquecida) fue de $12,6 \mu \mathrm{g} \cdot \mathrm{g}^{-1} \mathrm{Zn}$ (Tabla 3).

De la misma forma, si se compara el porcentaje de $\mathrm{Zn}$ asociado a la materia orgánica en ambas estaciones (Tabla 3), se observa que el Zn enlazado a materia orgánica es mayor en porcentaje y concentración en la estación enriquecida, lo que podría explicarse por la mayor cantidad de materia orgánica y la formación de complejos órgano metálicos. La materia orgánica total (MOT) corresponde aproximadamente a un $10 \%$ en la cabeza y $7 \%$ en la boca (Silva et al., 1998). Sin embargo, esto no podría explicar el incremento de $\mathrm{Zn}$ asociado a los oxihidróxidos y a la fracción residual. Esta concentración podría más bien atribuirse a la entrada adicional de óxidos y sulfuros de Zn de baja solubilidad, que pudieron formar parte de la fuga de los concentrados de $\mathrm{Zn}$ durante las actividades de carga de concentrados de $\mathrm{Zn}$ y Pb en Puerto Chacabuco.

La comparación del porcentaje de las fracciones (Fig. 2a), muestra que el $\mathrm{Zn}$ residual asociado a la parte estructural de la matriz alcanzó a un $85 \%$ en la Estación 78-a (boca del fiordo), contra un 60\% en la estación 82-a. Por otra parte, la concentración el $\mathrm{Zn}$ asociado al residual de la matriz fue un tercio 
Tabla 1. Resultado del análisis químico de los materiales de referencia certificado. La desviación estándar promedio se obtuvo de tres medidas independientes.

Table 1. Results of chemical analyses on certified reference materials. Standard deviation is obtained in three independent measurements.

\begin{tabular}{|ccccccc|}
\hline Metal & $\begin{array}{c}\text { Análisis } \\
\boldsymbol{\mu g} \cdot \mathbf{g}^{-1}\end{array}$ & $\begin{array}{c}\text { MESS-2 } \\
\boldsymbol{\mu g} \cdot \mathbf{g}^{-1}\end{array}$ & $\begin{array}{c}\text { Error relativo } \\
\boldsymbol{\%}\end{array}$ & $\begin{array}{c}\text { Análisis } \\
\boldsymbol{\mu g} \cdot \mathbf{L}^{-1}\end{array}$ & $\begin{array}{c}\text { CASS-3 } \\
\boldsymbol{\mu g} \cdot \mathbf{L}^{-\mathbf{1}}\end{array}$ & $\begin{array}{c}\text { Error relativo } \\
\boldsymbol{\%}\end{array}$ \\
\hline $\mathrm{Zn}$ & $188 \pm 15$ & $172 \pm 16$ & 9,3 & $1,63 \pm 0,05$ & $1,24 \pm 0,25$ & 9,3 \\
$\mathrm{Zn}^{*}$ & $157 \pm 1,8$ & $172 \pm 16$ & 8,7 & - & - & - \\
\hline
\end{tabular}

*AES -ICP (Ahumada, 1998)

Tabla 2. Determinación de Zn Total $\left(\mu \mathrm{g} \cdot \mathrm{g}^{-1}\right)$ en sedimentos marinos de Puerto Chacabuco. La desviación estándar promedio se obtuvo de tres medidas independientes.

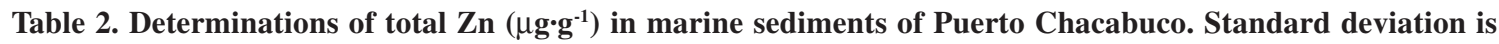
obtained in three independent measurements.

\begin{tabular}{|ccccc|}
\hline Localidad & Estación & Zn & Coeficiente de variación (\%) & Referencia \\
\hline Pto. Chacabuco & $82-\mathrm{a}$ & $168 \pm 15$ & 8,9 & $(1)$ \\
Pto. Chacabuco & 82 & $181 \pm 29$ & 16,0 & $(2)$ \\
Boca del Fiordo & $78-\mathrm{a}$ & $83,6 \pm 6$ & 7,2 & $(1)$ \\
Boca del Fiordo & 77 & $85,8 \pm 4$ & 4,7 & $(2)$ \\
\hline
\end{tabular}

(1) Este estudio (Espectrofotometría de Absorción Atómica con Tubo en la Llama).

(2) Ahumada (1998) (Espectrofotometría de Emisión Atómica con Inducción de Plasma).

(1) Present study (Atomic Absortion Spectrophotometry with Tube on Flame).

(2) Ahumada (1998) (Atomic Emission Spectrometry with Coupled Plasma Induction).

Tabla 3. Especiación de zinc en sedimentos superficiales del fiordo Aysén en noviembre de 2001. La desviación estándar promedio se obtuvo de tres medidas independientes.

Table 3. Zn speciation in the superficial sediments in Aysén Fjord in November 2001. Standard deviation is obtained in three independent measurements.

\begin{tabular}{|cc|ccccc|c|}
\cline { 3 - 7 } \multicolumn{1}{c|}{ Estación } & Unidad & Intercambiable & Carbonatos & $\begin{array}{c}\text { Óxidos } \\
\text { e hidróxidos }\end{array}$ & Materia & $\begin{array}{c}\text { Residual } \\
\text { orgánica }\end{array}$ & Total \\
\hline \multirow{2}{*}{$\mathbf{8 2}-\mathbf{a}$} & $\left(\mu \mathrm{g} \cdot \mathrm{g}^{-1}\right) \pm \sigma$ & $11,3 \pm 0,5$ & $1,3 \pm 0,2$ & 48,2 & $4,5 \pm 0,4$ & $99,7 \pm 13,9$ & $165 \pm 8$ \\
& $\%$ & 6,85 & 0,81 & 29,21 & 2,73 & 60,40 & \\
\multirow{7}{*}{$\mathbf{7 8 - a}$} & $\left(\mu \mathrm{g} \cdot \mathrm{g}^{-1}\right) \pm \sigma$ & $4,2 \pm 0,2$ & $3,2 \pm 0,2$ & 4,3 & $0,6 \pm 0,2$ & $71,3 \pm 4$ & 85,0 \\
& $\%$ & 4,9 & 3,8 & 5,1 & 0,7 & 83,9 & \\
\hline
\end{tabular}

de veces mayor. En la muestra de la Est. 82-a, la fracción asociada a oxihidróxidos fue seis veces superior en porcentaje y doce veces mayor en concentración. En cambio la fracción de Zn asociada a la materia orgánica fue mayor en porcentaje y concentración (cuatro y siete veces, respectivamente) en Puerto Chacabuco (Tabla 3). En general, el estudio de especiación de Zn mostró que en la Est. 82-a, el Zn de la fracción residual $>$ oxihidróxidos $>$ intercambiables $>$ materia orgánica $>$ carbonatos. En cambio, en la Est. 78-a, la fracción residual > oxihidróxidos 3 intercambiables $>$ carbonatos $>$ materia orgánica. Las diferencias entre las estaciones fueron mayores en concentración de $\mathrm{Zn}$ para los óxidos, hidróxidos y materia orgánica, aunque también presentó un incremento importante la fracción de residuales. En cambio, la estación de la boca favorecería la asociación de Zn a los carbonatos y proporcionalmente a los residuales.

La relación porcentual entre las especies $\mathrm{Zn}$ asociadas a los sitios estudiados, como comparación de procesos (porcentajes) para las diferentes frac- 
ciones encontradas fue, intercambiables : carbonatos : oxihidróxidos : materia orgánica : residuales = $1,37: 0,21: 5,73: 3,9: 0,71$. Por otra parte, la relación de concentración entre las fracciones para las dos estaciones analizadas (cabeza y boca del fiordo), fue: intercambiables: carbonatos: oxihidróxidos: materia orgánica: residuales $=2,7: 0,44: 11,2: 7,5$ $: 1,4$. Esto muestra una diferencia de 11,2 veces mayor de oxihidróxidos en Puerto Chacabuco y una relación inversa de carbonatos, con mayor concentración en la boca.

El análisis de los resultados de concentración, indica que existe una relación que duplicaría los valores de las fracciones en los sedimentos de Puerto Chacabuco, con excepción del Zn enlazado a los carbonatos. Se calculó una relación porcentual entre las fracciones para independizarlas del valor absoluto. La proporcionalidad de la relación de las fracciones entre los porcentajes y las relaciones de concentraciones absolutas se mantuvo. La relación porcentual permitió comparar las diferentes fracciones. Esta proporcionalidad, concuerda con los resultados encontrados por Howard \& Vandenbrink (1999), quienes observaron que el contenido de Zn está regulado por las condiciones ambientales y la contaminación, cuando esta es pequeña. De la misma forma, el incremento de $\mathrm{Zn}$ asociado a oxihidróxidos se debería al polvo fugitivo que escapa durante el embarque de concentrado de $\mathrm{Zn}$ (i.e. $\mathrm{ZnS}$ y $\mathrm{ZnO}$ ) y a su baja solubilización en el agua de mar (Kps < $\left.10^{-30}\right)$.

\section{CONCLUSIONES}

De acuerdo a los resultados encontrados, se puede concluir que:

1. Las variaciones en la concentración de las fracciones de Zn muestran cambios que pueden atribuirse a condiciones específicas de los sitios: carbonatos (Est. 78-a), materia orgánica (Est. 82-a) y derivados de su condición natural.

2. Existen cambios de concentración que no pueden explicarse tan claramente. En la cabeza la concentración de oxihidróxidos fue 11 veces mayor en valor absoluto y mayor también en porcentaje (i.e. 5,7 veces) y la fracción de $\mathrm{Zn}$ residual, mayor en valor absoluto es menor en porcentaje, pudiendo ser atribuido a la actividad antrópica desarrollada del área.

3. La mayor biodisponibilidad de Zn se observó en la zona enriquecida, donde podría ocurrir el in- tercambio entre el sedimento y la columna de agua. Sin embargo, al cuantificar estas fracciones, no se observa un cambio de concentraciones que pudiera generar un riesgo ambiental para los organismos de la zona.

\section{AGRADECIMIENTOS}

Los autores agradecen al Comité Oceanográfico Nacional que financió el Proyecto "Estudio geoquímico de metales y fuentes de enriquecimiento de $\mathrm{Pb}$ y $\mathrm{Zn}$ en sedimentos marinos de origen fluvio-glacial" en el programa Cimar 7 Fiordos con el aporte del Ministerio de Hacienda de Chile. Al Proyecto MECESUP USC 9901. A Ruby Riveros por el ataque y preparación de las muestras de sedimento para análisis. Además, agradecen a los revisores anónimos que con sus críticas permitieron mejorar este trabajo.

\section{REFERENCIAS}

Ahumada, R. 1998. Metales traza contenidos en los sedimentos del seno Aysén: Línea base y alteraciones antrópicas. Cienc. Tecnol. Mar, 21: 75-88.

Ahumada R. \& S. Contreras. 1999. Contenido de metales ( $\mathrm{Ba}, \mathrm{Cd}, \mathrm{Co}, \mathrm{Cr}, \mathrm{Cu}, \mathrm{Ni}, \mathrm{Pb}, \mathrm{Sr}, \mathrm{V}$ y Zn) en sedimentos de los fiordos adyacentes a Campo de Hielo Sur y Canal Concepción. Cienc. Tecnol. Mar, 22: 24-41.

Ahumada, R., A. Rudolph \& S. Contreras. 2002. Contenido de metales ( $\mathrm{Ba}, \mathrm{Cd}, \mathrm{Co}, \mathrm{Cr}, \mathrm{Cu}, \mathrm{Ni}, \mathrm{Pb}$, $\mathrm{Sr}, \mathrm{V}$ y $\mathrm{Zn}$ ) en sedimentos de los fiordos Patagónicos $\left(52^{\circ}-56^{\circ} \mathrm{S}\right)$, Chile. Cienc. Tecnol. Mar, 25(2): 77-86.

Araya, J.F. 1997. Perfiles geomorfológicos de los fiordos y depresión longitudinal de norpatagonia. Cienc. Tecnol. Mar, 20: 3-86.

EURACHEM, 2001. Quantifying uncertainly in analytical measurement. EUROCHEM/CITAC. Guide CG 4: 91 pp.

Davidson, C.M., R.P. Thomas, S.E. McVey, R. Perala, D. Littlejohn \& A.U. Ure. 1994. Evaluation of sequential extraction procedure for speciation of heavy metals in sediments. Anal. Chim. Acta, 291: 277-286. 
Howard, J.L. \& G. Sledzinski. 1996. Geochemical behavior of lead in an altisol an ultisol at high levels of contamination. J. Soil Contam., 5: 61-81.

Howard, J.L. \& W.J. Vandenbrink. 1999. Sequential extraction analysis of heavy metals in sediments of variable composition using nitrilotriacetic acid to counteract resortion. Environ. Pollut., 106: 285292.

Kim, D. \& J.E. Fergunsson. 1991. Effectiveness of commonly used sequential extraction technique in determining speciation of cadmium in soils. Sci. Total Environ., 105: 191-209.

Libes, S.M. 1992. An introduction to marine biogeochemistry. John Wiley \& Sons, New York, 734 pp.

Luoma, S.N. \& J.A. Davis. 1983. Requirements for modeling trace metal partitioning in oxidized estuarine sediments. Mar. Chem., 12: 159-181.

Martin, J.M., P. Nirel \& A.J. Thomas. 1987. Sequential extraction techniques: promises and problems. Mar. Chem., 22: 313-341.

Neira, J. \& H. Berndt. 2000. Determination of Cd and $\mathrm{Pb}$ at $\mu \mathrm{g} / \mathrm{L}$ levels by HHPN-beam injection flame furnace-AAS. Fresenius Z. Anal. Chem., 368: 649-655.

Pickard, G.L. 1973. Some physical oceanographic features of inlets of Chile. J. Fish. Bd. Canada, 28: 1077-1106.

Pineda, V., X. Contardo, G. Alfaro \& S. Helle. 2002. Caracterización textural, mineralógica y geoquímica de sedimentos del canal Beagle, bahía Nassau. Magallanes, Chile. Crucero Cimar Fiordo 3. Cienc. Tecnol. Mar, 25(1): 5-21.

Recibido: 11 marzo 2003; Aceptado: 7 enero 2004
Rivera-Duarte, I. \& A.R. Flegal. 1997. Pore water silver concentration gradient and benthic fluxes from contaminated sediments of San Francisco Bay, California, USA. Mar. Chem., 56: 15-26.

Silva, N., C. Calvete \& H. Sievers. 1997. Características oceanográficas físicas y químicas de canales australes chilenos entre Puerto Montt y laguna San Rafael (Crucero Cimar Fiordo 1). Cienc. Tecnol. Mar, 20: 23-106.

Silva, N., J. Maturana, J.I. Sepúlveda \& R. Ahumada. 1998. Materia orgánica, $C$ y N, su distribución y estequiometría, en sedimentos superficiales de la región norte de los fiordos y canales australes de Chile. (Crucero Cimar-Fiordo I). Cienc. Tecnol. Mar, 21: 49-74.

Tessier, A. \& P.G.C. Campbell. 1988. Partitioning of trace metals in sediments. En: J. Kramer \& H. Allen (eds.). Metal speciations: theory, analysis and application. Lewis Publishers, Chelsea, pp.183199.

Tessier, A. 1999. Chemical speciation of metals and bioavailability. Proceedings of $8^{\text {th }}$ European Research Conference. Conférencier invité. Carfagnana, Italie, 298 pp.

Warnken, K.W., G.A. Gill, L.L. Griffin \& P.H. Santschi. 2001. Sediment-water exchange of Mn, $\mathrm{Fe}, \mathrm{Ni}$ and $\mathrm{Zn}$ in Galveston Bay, Texas. Mar. Chem., 73: 215-231.

Wen, L.-S., P. Santschi, G. Gill \& C. Paternostro. 1999. Estuarine trace metal distributions in Galveston Bay: importance of colloidal form in speciation of dissolved phase. Mar. Chem., 63: 185212. 


\section{ANEXO 1}

Descripción del esquema de ataque diferencial aplicado a 0,5 g de sedimentos marinos secos (Tessier \& Campbell, 1988).

1. Primer extracto (intercambiables). Lixiviación con cloruro de magnesio

$8 \mathrm{~mL}$ de cloruro de magnesio $1 \mathrm{M}$ a pH 7 (ajustar $\mathrm{pH}$ con $\mathrm{NaOH} 0,1 \mathrm{M}$ ) + agitación continua a temperatura ambiente por $1 \mathrm{~h}$.

2. Segundo extracto (carbonatos). Lixiviación con acetato de sodio

$8 \mathrm{~mL} \mathrm{NaOAc} 1 \mathrm{M}$ a pH 5 (ajustar pH con $\mathrm{HOAc}_{(\mathrm{c})}$ ) + agitación continua a temperatura ambiente por $5 \mathrm{~h}$.

3. Tercer extracto (óxidos - hidróxidos)

$20 \mathrm{~mL} \mathrm{Na}_{2} \mathrm{~S}_{2} \mathrm{O}_{4} 0,3 \mathrm{M}+20 \mathrm{~mL}$ Na-citrato $0,175 \mathrm{M}+20 \mathrm{~mL}$ H-citrato $0,025 \mathrm{M}+$ agitación ocasional a $96^{\circ} \mathrm{C}$ por $5 \mathrm{~h}$.

\section{Cuarto extracto (materia orgánica)}

$3 \mathrm{~mL} \mathrm{HNO}_{3} 0,02 \mathrm{M}+5 \mathrm{~mL} \mathrm{H}_{2} \mathrm{O}_{2} 30 \%$ a pH 2 (ajustar $\mathrm{pH}$ con $\mathrm{HNO}_{3} 0,2 \mathrm{M}$ ). Extracción a $85^{\circ} \mathrm{C}$ por $12 \mathrm{~h}$, con agitación ocasional. Enfriar, luego agregar $5 \mathrm{~mL} \mathrm{NH} \mathrm{OAc}_{4}, 2 \mathrm{M}$ en $\mathrm{HNO}_{3} 20 \%$ v/v. Dilución a 10 $\mathrm{mL}$, agitación continua por $30 \mathrm{~min}$.

5. Quinto extracto (residual)

$10 \mathrm{~mL} \mathrm{HF}_{(\mathrm{c})}+2 \mathrm{~mL} \mathrm{HClO}_{4(\mathrm{c})}$. Extracción hasta sequedad. Luego $10 \mathrm{~mL}$ de $\mathrm{HF}_{(\mathrm{c})}+1 \mathrm{~mL} \mathrm{HClO}_{4(\mathrm{c})}$ relación 5:1. Extracción hasta desaparición de vapores blancos. Dilución con $10 \mathrm{~mL}$ de $\mathrm{HCl} 12 \mathrm{~N}$ y aforar a 25 $\mathrm{mL}$ con agua milli $\mathrm{Q}$. 